\title{
Cation-Dependent Hierarchical Assembly of U60 Nanoclusters into Blackberries Imaged via Cryogenic Transmission Electron Microscopy
}

\author{
Jennifer A. Soltis ${ }^{1}$, Christine M. Wallace ${ }^{2}$, R. Lee Penn ${ }^{1}$, and Peter C. Burns ${ }^{2,3}$ \\ 1. Department of Chemistry, University of Minnesota, Minneapolis, Minnesota, United States \\ 2. Department of Civil \& Environmental Engineering \& Earth Sciences, University of Notre Dame, \\ Notre Dame, Indiana, United States \\ 3. Department of Chemistry and Biochemistry, University of Notre Dame, Notre Dame, Indiana, United \\ States
}

The self-assembly of polyoxometalate nanoclusters into secondary and tertiary superstructures is of great interest to many fields, including sensing, catalysis, and biomedicine. Polyoxometalates often have unique physical properties that further heightens this appeal. When in the solution phase, these structures often have an overall negative charge and behave as dissolved species, leading to their description as macroanions. Their anionic character provides a facile route by which to tailor self-assembly behavior through the addition of positively charged species.

U60 is a uranyl peroxide nanocluster composed of 60 uranyl peroxide hydroxide hexagonal bipyramids in Buckminster fullerene topology [1]. U60, like many other polyoxometalate nanoclusters, has been shown to self-assemble into hollow spherical structures referred to as "blackberries" [2]. These larger structures are only stable while in the solution phase, making ex situ characterization particularly difficult. Two of the specific challenges faced when trying to image U60 nanoclusters are the rapid rate of reduction of U60 to uranium oxide phases when exposed to the electron beam of an electron microscope and the collapse of U60 blackberries upon drying in conventional transmission electron microscopy (TEM) specimen preparation. We have overcome this second limitation through the use of cryogenic (cryo) TEM to image U60 nanoclusters, blackberries, and tertiary structures in their in situ conformation.

Cryo-TEM is a powerful tool that enables high resolution imaging of samples that would otherwise not survive the high vacuum environment found in a TEM, including samples that are altered or damaged by drying. By rapidly cooling a specimen in liquid ethane, the solvent component is vitrified into an amorphous, electron transparent solid. This process preserves the in situ conformation of the sample of interest, be it nanoparticles, micelles, viruses, or proteins.

Here, cryo-TEM was used to investigate the cation-driven self-assembly behavior of U60 nanoclusters in solution. Nitrate salts of sodium, potassium, calcium, and magnesium were added to solutions of U60 nanoclusters, and aliquots were vitrified in liquid ethane at various times after the addition of the salts. All further storage and handling of the vitrified specimens occurred under liquid nitrogen to maintain cryogenic conditions. Individual specimens were loaded into a Gatan 626 cryo-transfer holder and imaged under low-dose conditions on an FEI Tecnai $\mathrm{G}^{2}$ F30 field-emission gun TEM operated at 300 $\mathrm{kV}$. Ultra-small and small angle X-ray scattering measurements were performed on beamline 15-ID at the Advanced Photon Source [3].

The kinetics of self-assembly and the morphology of the secondary and tertiary structures produced are highly sensitive to the valence state and identity of the added cation. The addition of the divalent cations 
$\mathrm{Mg}^{2+}$ and $\mathrm{Ca}^{2+}$ induced the formation of hollow, spherical blackberries - a secondary level of assembly. Figure 1a shows a cryo-TEM image of a U60 solution vitrified 34 minutes after the addition of a calcium nitrate solution. Individual, ca. 2.5-nm U60 clusters can be seen assembled into a ca. 30-nm blackberry. The addition of the monovalent cations $\mathrm{Na}^{+}$and $\mathrm{K}^{+}$induced the formation of fractal-like tertiary structures that appear to be composed of many blackberry subunits. Figure $1 \mathrm{~b}$ shows a cryoTEM image of a U60 solution vitrified 15 minutes after the addition of a potassium nitrate solution. Individual U60 clusters are still discretely visible as members of the blackberries and fractal-like structure that represent the secondary and tertiary levels of self-assembly, respectively [3].

We present here the first cryo-TEM imaging of polyoxometalate superstructures in situ. We show that the kinetics of assembly and final morphology of U60 superstructures is dependent on the identity of the added cation and propose a route of hierarchical assembly in the formation of these structures.[4]

\section{References:}

[1] J Qiu and PC Burns, Chemical Reviews 113 (2013), p. 1097-1120.

[2] T Liu, Langmuir 26 (2009), p. 9202-9213.

[3] JA Soltis et al, Journal of the American Chemical Society 138 (2016), p. 191-198.

[4] We thank Jan Ilavsky at the Advanced Photon Source, Argonne National Laboratory, for his assistance with pinhole SAXS and USAXS measurements. Participation of JAS and RLP was funded by the National Science Foundation (\#0957696) and the University of Minnesota IPrime Nanostructural Materials and Processes Program. Participation of CMW and PCB was funded by the Office of Basic Energy Sciences of the U.S. Department of Energy as part of the Materials Science of Actinides Energy Frontiers Research Center (DE-SC0001089). Parts of this work were carried out in the Characterization Facility, University of Minnesota, a member of the NSF-funded Materials Research Facilities Network (www.mrfn.org) via the MRSEC program.
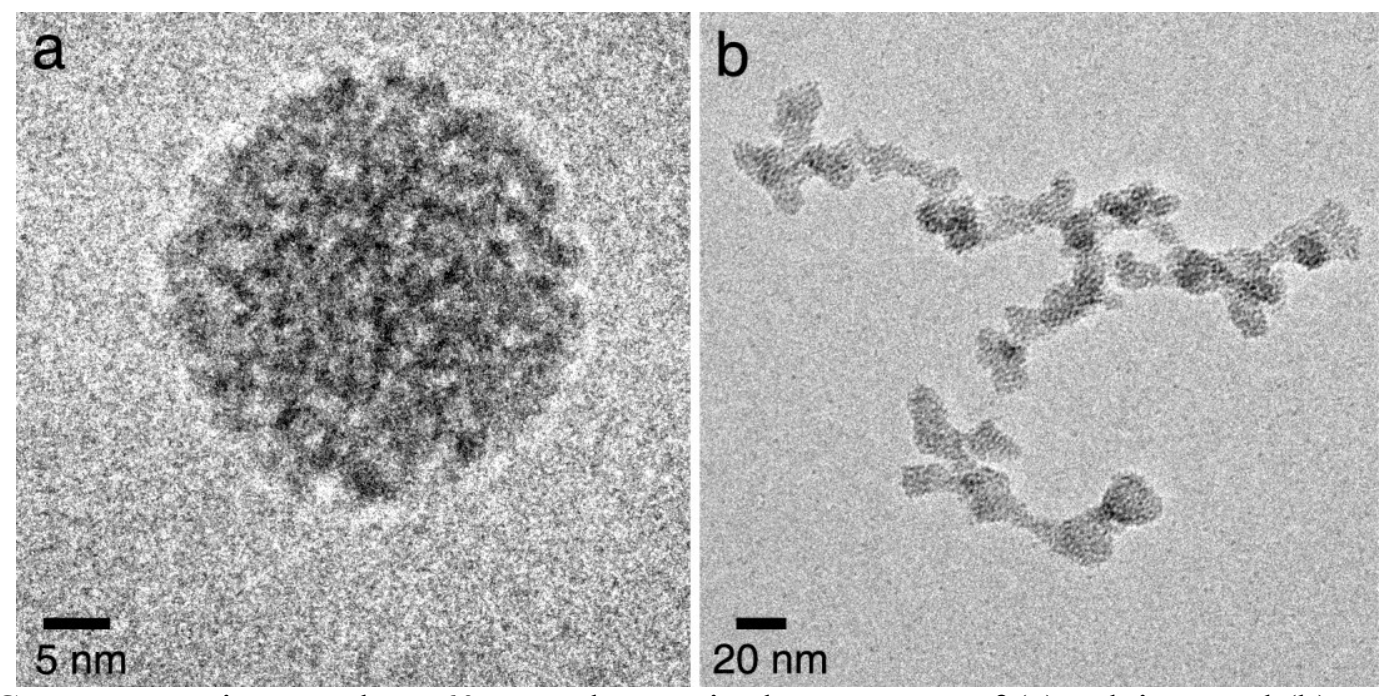

Figure 1. Cryo-TEM micrographs U60 nanoclusters in the presence of (a) calcium and (b) potassium nitrate. (a) was vitrified 34 minutes after the addition of the nitrate salt to the U60 solution. (b) was vitrified 15 minutes after the addition of the nitrate salt to the U60 solution. 